\title{
DR-SIP: Protocols for Higher Order Structure Modeling with Distance Restraints- and Cyclic Symmetry-Imposed Packing
}

Justin Chan ${ }^{1,2}$, Jinhao Zou ${ }^{1}$, Chi-Hong Chang Chien ${ }^{1}$, Rong-Long Pan ${ }^{1}$, Lee-Wei Yang ${ }^{1,2,3, *}$

${ }^{1}$ Institute of Bioinformatics and Structural Biology, National Tsing Hua University, Hsinchu, Taiwan

${ }^{2}$ Bioinformatics Program, Taiwan International Graduate Program, Institute of Information Sciences, Academia Sinica, Taipei, Taiwan

${ }^{3}$ Physics Division, National Center for Theoretical Sciences, National Tsing Hua University, Hsinchu, Taiwan

*Correspondence: 1wyang@life.nthu.edu.tw 


\begin{abstract}
Motivation

Quaternary structure determination for proteins is difficult especially for transmembrane proteins. Even if the monomeric constituents of complexes have been experimentally resolved, computational prediction of quaternary structures is a challenging task particularly for higher order complexes. It is essential to have a reliable computational protocol to predict quaternary structures of both transmembrane and soluble proteins leveraging experimentally determined distance restraints and/or cyclic symmetry $\left(\mathrm{C}_{n}\right.$ symmetry) found in most homo-oligomeric transmembrane proteins.
\end{abstract}

\title{
Results
}

We survey $115 \mathrm{X}$-ray crystallographically solved structures of homo-oligomeric transmembrane proteins (HoTPs) to discover that $90 \%$ of them are $\mathrm{C}_{n}$ symmetric. Given the prevalence of $\mathrm{C}_{n}$ symmetric HoTPs and the benefits of incorporating geometry restraints in aiding quaternary structure determination, we introduce two new filters, the distance-restraints (DR) filter and the Symmetry-Imposed Packing (SIP) filter which takes advantage of the statistically derived tilt angle cutoff and the $\mathrm{C}_{\mathrm{n}}$ symmetry of HoTPs without prior knowledge of the number (" $n$ ") of monomers. Using only the geometrical filter, SIP, near-native poses of the 115 HoTPs can be correctly identified in the top-5 for $52 \%$ of all cases, or $49 \%$ among the HoTPs having an $n>2(\sim 60 \%$ of the dataset), while ZDOCK alone returns $41 \%$ and $24 \%$, respectively. Applying only SIP to three HoTPs with distance restraints, the near-native poses for two HoTPs are ranked $1^{\text {st }}$ and the other $7^{\text {th }}$ among 54,000 possible decoys. With both filters, the two remain $1^{\text {st }}$ while the other improved to $2^{\text {nd }}$. While a soluble system with distance restraints is recovered at the $1^{\text {st }}$-ranked pose by applying only DR.

\section{Availability and Implementation https://github.com/capslockwizard/drsip}

\section{Supplementary information}

Supplementary methods and results are available. 


\section{INTRODUCTION}

The quaternary structure of proteins provides atomistic details which can be used to study the mechanisms underlying the function of these proteins. A large subset of these protein complexes are transmembrane proteins which constitute 20-30\% of the proteome (Fagerberg et al., 2010) and are the second most common drug targets (Rask-Andersen et al., 2014), after enzymes. A survey on RCSB PDB (Rose et al., 2017) shows that about $66 \%$ of $\alpha$-helical transmembrane proteins are homo-oligomeric transmembrane proteins (HoTPs), while 92\% of HoTPs are cyclic $\left(\mathrm{C}_{n}\right)$ symmetric (Fig. 1).

Experimentally solving the ternary and quaternary structures of transmembrane proteins is challenging. The general difficulty is in purifying proteins in sufficient quantity and purity. For Xray crystallography, a major challenge after obtaining proteins is in screening for the optimal conditions to reconstitute and crystalize the proteins (Moraes et al., 2014). On the other hand, solution NMR is limited to protein complexes with a high tumbling rate (Liang and Tamm, 2016) which limits the size of the complex while solid-state NMR is limited by spectral crowding, which is further aggravated by large complexes (Liang and Tamm, 2016).

Computational methods such as homology docking has been applied to predict quaternary structures which are evolutionarily conserved (Levy et al., 2008). These methods (Szilagyi and Zhang, 2014; Bertoni et al., 2017) identify structurally solved homologous protein complexes, not just constituent proteins, as the templates for the homology modelling. However, to predict higher order structures for proteins that lack any structural template of homologs, physiochemically driven molecular docking could be the only option to go about.

Molecular docking is a method that usually generates tens of thousands of orientations (poses) of two proteins bound to one another which are then rank-ordered by a scoring function that evaluates the fitness (or "energy") of these poses (Huang, 2014). Identifying near-native poses among the $>10,000$ generated poses is difficult because of the efficient sampling in molecular docking comes at the expense of accurately evaluating the solvent and entropy's energy (Brooijmans and Kuntz, 2003). Common docking packages include ZDOCK (Pierce et al., 2011), ClusPro (Kozakov et al., 2017), GRAMM-X (Tovchigrechko and Vakser, 2006), RosettaDock (Chaudhury and Gray, 2008) and HADDOCK (van Zundert et al., 2016), where the latter two allow for experimentally determined restraints or prior knowledge of pairwise distances to constraint and filter the docking results.

Predicting interactions between membrane proteins, DOCK/PIERR (Viswanath et al., 2015), ROSETTA:MPdock (Alford et al., 2015) and Memdock (Hurwitz et al., 2016) generate poses whose transmembrane helices in the constituent monomers are embedded in the membrane bilayer, which enriches the near-native poses among the decoys. There are also docking packages that specifically perform $\mathrm{C}_{\mathrm{n}}$ symmetric docking such as SymmDock (Schneidman-Duhovny et al., 2005), ClusPro (Kozakov et al., 2017), M-ZDOCK (Pierce et al., 2005), ROSETTA:MPsymdock (Alford et al., 2015) and HADDOCK (van Zundert et al., 2016). Although these methods are successful at predicting the native complexes, they require prior knowledge of the number of constituent monomers in the complex, or the "order of symmetry".

Integrative approaches, combining multiple sources of experimental data with computational methods, have been used to confine the search space during generation of poses or to filter out the 
poses that are incompatible with the data thereby enriching the near-native ones among the docking decoys (Alber et al., 2008). Biochemically and biophysically determined distance restraints include but are not limited to the data obtained from mutagenesis, cross-linking, hydrogen/deuterium exchange, electron microscopy (Mitra and Frank, 2006), small-angle X-ray scattering (Yamagata and Tainer, 2007), NMR chemical shift perturbations (Gupta et al., 2013; Chang et al., 2016; Khan et al., 2018) and single molecule resonance energy transfer (smFRET) (Dimura et al., 2016; Choi et al., 2010; Muschielok et al., 2008), which have been used to predict quaternary structures (Alber et al., 2008).

Among these biophysical methods, smFRET measures the distance between two fluorophore dyes acting as labels attached to residues in proteins or nucleotides to study protein folding, proteinprotein interaction and protein-DNA/RNA interaction (Sasmal et al., 2016). However, these distances suffer from uncertainties due to the effect of the local environment on the dye pairs (Dimura et al., 2016; Kalinin et al., 2012; Muschielok et al., 2008).

In this study, we introduce two new docking protocols including the two new filters, the Distance Restraints (DR) filter and Symmetry-Imposed Packing (SIP) filter, to facilitate quaternary structure determination for soluble and transmembrane proteins. Here the "DR" filter requires experimentally measured distance restraints but the geometric filter, SIP, requires no experimental input. The SIP filter removes the docking poses (homo-dimer) that deviate significantly from its ideal $C_{n}$ symmetry without prior knowledge of the order of symmetry (the $n$ in $C_{n}$ ) and those having large tilt angles. Given the uncertainties of the absolute distances obtained from smFRET, the DR filter uses the agreement in the relative ranking of these distances by correlating the smFRETmeasured intramolecular distances and their counterparts in the docking poses. The cutoffs for these filters are derived from the statistical analyses of a dataset containing the X-ray structures of $118 \alpha$-helical HoTPs (see Results). Evaluating only the SIP filter without distance restraints on all $115 \mathrm{C}_{\mathrm{n}}$ symmetric HoTPs from the dataset shows the near-native poses in $64 \%$ of HoTPs can be recovered within the top-10 results starting from the 54,000 poses generated by ZDOCK, as compared to the $45 \%$ recovered by ZDOCK alone. For the $59 \%$ of HoTPs that are larger than dimers $(n>2)$, SIP recovers $57 \%$ of the native poses in the top-10 while ZDOCK alone merely recovers $28 \%$. Furthermore, for four systems (soluble: Syt1-SNARE complex, three HoTPs: MscL, $\mathrm{VrH}^{+}$-PPase and $\mathrm{Ct} \mathrm{H}^{+}$-PPase) with experimentally determined distance restraints, the DR-SIP filters return a near-native pose in the top rank for three systems and $2^{\text {nd }}$-rank for MscL. Without the DR filter, a near-native pose for MscL is $7^{\text {th }}$-ranked while the other two HoTPs are still topranked. Other than ZDOCK, DR-SIP is applied to GRAMM-X's results and the near-native poses are enriched up to $\sim 167$-fold such that they make up one out of every three remaining poses. 


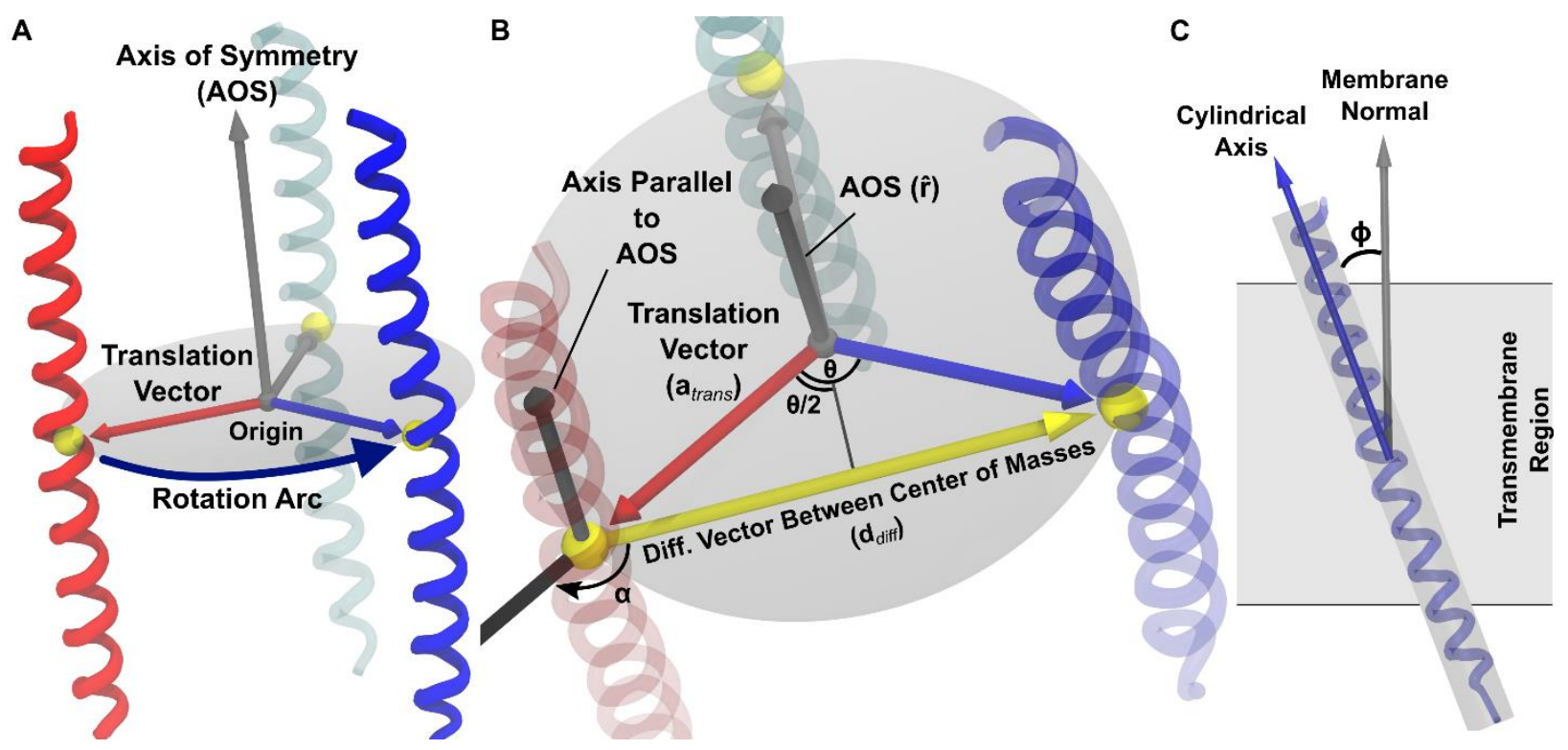

Fig. 1. $C_{n}$ symmetry and tilt angle of HoTPs. (A) Three helices with $C_{3}$ symmetry about the axis of rotation/symmetry (AOS). (B) The translation vector ( $\mathbf{a}_{\text {trans }}$ ) can be expressed as a function of angle of rotation $(\theta)$ and difference vector between the centers of mass of the two monomers $\left(\mathbf{d}_{\text {diff }}\right)$ such that $\mathbf{a}_{\text {trans }}$ is obtained by rotating $\mathbf{d}_{\text {diff }}$ by $\boldsymbol{\alpha}=90^{\circ}+\theta / 2$ degree about the $\operatorname{AOS}(\hat{\mathbf{r}})$ and then rescaled to $\| \mathbf{d}_{d i f f} / /(2 \sin (\theta / 2))$ for its magnitude. $(\mathbf{C})$ Tilt angle $(\phi)$ is the acute angle between the cylindrical axis and membrane normal (assumed to be parallel to AOS). 


\section{METHODS}

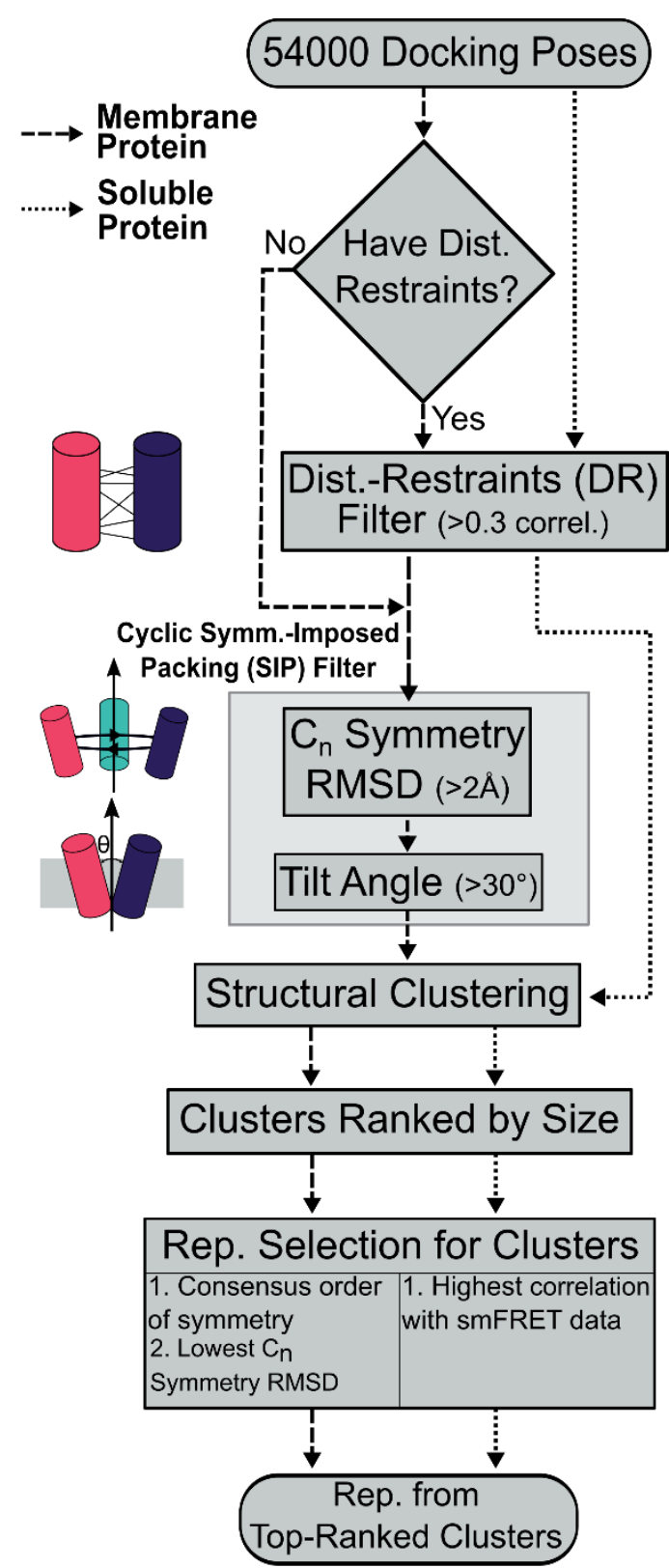

Fig. 2. Flowchart of DR-SIP's membrane and soluble protein docking protocols. Each cluster is represented by one member of the cluster. The final results are the representatives ranked based on the size of the clusters.

\section{Docking Protocols}

DR-SIP contains two docking protocols (Fig. 2). The first protocol is for predicting the quaternary structures of HoTPs (dashed line in Fig. 2) and the other for soluble proteins (dotted line in Fig. 2). The docking protocols make use of the DR and SIP filters. The SIP filter (see below and Supplementary Methods) consists of the $\mathrm{C}_{\mathrm{n}}$ symmetry root-mean squared deviation (RMSD) and the tilt angle criteria.

For every system, 54,000 docking poses are generated using ZDOCK 3.0.2 (Pierce et al., 2011) with $6^{\circ}$ rotational sampling (dense sampling).

$\mathrm{C}_{\mathrm{n}}$ symmetry root-mean-squared deviation $\left(\mathrm{C}_{\mathrm{n}} \mathrm{RMSD}\right)$ measures the current pose's deviation from its closest ideal $\mathrm{C}_{\mathrm{n}}$ symmetric pose (see Methods). The axis and order of symmetry $(n)$ are estimated from each docking pose containing two monomers and used to generate the ideal $\mathrm{C}_{n}$ symmetric pose. Based on our statistical analysis (see Figs. 3C and 4), non- $\mathrm{C}_{n}$ symmetric poses with $>2 \AA C_{n}$ RMSD are removed.

While, tilt angle is the acute angle between the membrane's normal and the direction the monomer spans the membrane (Fig. 1C). Assuming the membrane normal is the axis of symmetry (AOS) of HoTPs, poses having tilt angles $>35^{\circ}$ are removed in accordance with the observed HoTP tilt angle distribution (see Fig. 3F).

The distance-restraints (DR) filter removes non-native poses that are incompatible with the experimentally measured constraints (herein smFRET-characterized distances) where relative distances rather than absolute distances are used. This is measured by the Pearson and Spearman correlations between the smFRET-measured distances and the distances of the corresponding labeled residues (between $\mathrm{C} \alpha$ atoms) in the docking pose. Poses are removed if they have $\leq 0.3$ correlation.

After applying the filters, the remaining poses are clustered using agglomerative clustering with the average linkage criteria (Manning et al., 2008), see Supplementary Methods for details. RMSD is used to measure the difference/distance between all pairs of poses. A cutoff of $12 \AA$ is 
employed and clusters with fewer than three poses are removed. The clusters for proteins are ranked based on their sizes in descending order.

For HoTPs, each cluster is assigned a consensus order of symmetry $(n)$, such that most members in the cluster have this ' $n$ '. The representative pose of each cluster must have the consensus order of symmetry and is the closest to the ideal $C_{n}$ symmetry as measured by the smallest $C_{n}$ symmetry RMSD.

For soluble proteins, the representative pose of each cluster has the highest Spearman correlation with the distance restraints data (smFRET data). If there are $\geq 2$ poses with the same Spearman correlation, the one with the higher Pearson correlation is chosen as the representative.

The final results returned to the user are the representative poses from the top clusters. Details of the filters and clustering method are in the Supplementary Methods.

\section{Extracting $C_{n}$ Symmetry Parameters from an Ideal $C_{n}$ Symmetric Pose}

As explained before, also true for this and the next sections, each ideal $C_{n}$ symmetric HoTP pose contains two identical and neighboring monomers (the blue and red monomers of the $\mathrm{C}_{3}$ complex in Fig. 1A) that is a part of $\left(C_{n>2}\right)$ or is the full $\left(C_{2}\right)$ protein complex. The number of monomers in a complex can be determined by dividing $2 \pi$ with the angle of rotation between these two monomers (see below).

The two monomers, A and A', are related by

$$
\mathbf{A}^{\prime}=\mathbf{R}(\mathbf{A})=\mathbf{R}\left(\mathbf{A}_{0}+\mathbf{A}_{\text {trans }}\right)=\mathbf{R} \mathbf{A}_{0}+\mathbf{R} \mathbf{A}_{\text {trans }}=\mathbf{A}_{0}^{\prime}+\mathbf{R} \mathbf{A}_{\text {trans }}
$$

where $\mathbf{A}$ and $\mathbf{A}^{\prime}$ are $3 \times M$ coordinate matrices of $M$ atoms for A and A', respectively. $\mathbf{R}$ is a rotation matrix that rotates about the AOS by $2 \pi / n \operatorname{rad}(n$ is the order of symmetry). In Equation (1), the $\mathrm{COM}$ of the n-mer complex is assumed to be at the origin (e.g. the center of the trimer in Fig 1A). When the COM of A and A' are placed at the origin, their coordinate matrices are $\mathbf{A}_{0}$ and $\mathbf{A}^{\prime}$, respectively. Let $\mathbf{A}_{\text {trans }}=\mathbf{a}_{\text {trans }}[1,1, \ldots 1]_{1 \times \mathrm{M}}$ where $\mathbf{a}_{\text {trans }}$ is the $3 \times 1$ vector pointing from the origin to the $\mathrm{COM}$ of $\mathrm{A}$.

The docking pose provides $\mathbf{A}$ and $\mathbf{A}^{\prime}$ which are used to compute the AOS $(\hat{\mathbf{r}})$, angle of rotation $\left(\theta_{r}\right)$, and the translation vector $\left(\mathbf{a}_{\text {trans }}\right)$. These three parameters define the $\mathrm{C}_{\mathrm{n}}$ symmetric relationship between $A$ and $A^{\prime}$ which can be used to reconstruct the n-mer complex.

$\hat{\mathbf{r}}$ and $\theta_{r}$ can be obtained from $\mathbf{R}$. As for $\mathbf{R}$, from Equation (1), $\mathbf{A}^{\prime}{ }_{0}=\mathbf{R A}_{0}$, the Kabsch algorithm is used to find an $\mathbf{R}$ that minimizes the RMSD between $\mathbf{A}^{\prime}{ }_{0}$ and $\mathbf{R} \mathbf{A}_{0}$ (Kabsch, 1976; Cock et al., 2009).

Given $\mathbf{R}, \hat{\mathbf{r}}$ is the eigenvector associated with the unity eigenvalue such that $\mathbf{R} \hat{\mathbf{r}}=1 \hat{\mathbf{r}}$, because the AOS is invariant when operated on by $\mathbf{R}$.

As for $\theta_{r}$, it can be found by extracting $\cos \theta_{r}$ and $\sin \theta_{r}$ from $\mathbf{R}$ which can be formulated as (Jia, 2017): 


$$
\mathbf{R}=\left[\begin{array}{ccc}
\cos \theta_{r}+r_{x}^{2}\left(1-\cos \theta_{r}\right) & r_{x} r_{y}\left(1-\cos \theta_{r}\right)-r_{z} \sin \theta_{r} & r_{x} r_{z}\left(1-\cos \theta_{r}\right)+r_{y} \sin \theta_{r} \\
r_{x} r_{y}\left(1-\cos \theta_{r}\right)+r_{z} \sin \theta_{r} & \cos \theta_{r}+r_{y}^{2}\left(1-\cos \theta_{r}\right) & r_{y} r_{z}\left(1-\cos \theta_{r}\right)-r_{x} \sin \theta_{r} \\
r_{x} r_{z}\left(1-\cos \theta_{r}\right)-r_{y} \sin \theta_{r} & r_{y} r_{z}\left(1-\cos \theta_{r}\right)+r_{x} \sin \theta_{r} & \cos \theta_{r}+r_{z}^{2}\left(1-\cos \theta_{r}\right)
\end{array}\right]
$$

where $\theta_{r}$ is the rotation angle and $\hat{\mathbf{r}}=\left(r_{x}, r_{y}, r_{z}\right)$ is the AOS (unit vector). The trace of $\mathbf{R}$ is $1+2 \cos$ $\theta_{r}$. Thus $\cos \theta_{r}$ can be obtained.

While, $\sin \theta_{r}$ is obtained as follows

$$
\mathbf{R}-\mathbf{R}^{\boldsymbol{T}}=\left[\begin{array}{ccc}
0 & -2 r_{z} \sin \theta_{r} & 2 r_{y} \sin \theta_{r} \\
2 r_{z} \sin \theta_{r} & 0 & -2 r_{x} \sin \theta_{r} \\
-2 r_{y} \sin \theta_{r} & 2 r_{x} \sin \theta_{r} & 0
\end{array}\right]
$$

where $2 r_{x} \sin \theta_{r}+2 r_{y} \sin \theta_{r}+2 r_{z} \sin \theta_{r}=2 \sin \theta_{r}\left(r_{x}+r_{y}+r_{z}\right)$. With $\hat{\mathbf{r}}$, we solve for $\sin \theta_{r}$.

Finally, $\theta_{r}=\operatorname{sign}\left(\sin \theta_{r}\right) \arccos \theta_{r}$. Alternatively, $\theta_{r}$ can be computed using the atan2 function which is implemented in most mathematical software libraries (van der Walt et al., 2011).

$\mathbf{a}_{\text {trans }}$ is obtained by rotating $\mathbf{d}_{\text {diff }}$ about $\hat{\mathbf{r}}$ by $\alpha$ radians (Fig. 1B), which is then rescaled to $\left\|\mathbf{a}_{\text {trans }}\right\|$ based on its trigonometric relationship with $\mathbf{d}_{\text {diff }}$

$$
\begin{gathered}
\left\|\mathbf{a}_{\text {trans }}\right\|=\left|\frac{\left\|\mathbf{d}_{\text {diff }}\right\|}{2 \sin \left(\frac{\theta_{r}}{2}\right)}\right| \\
\mathbf{a}_{\text {trans }}=\mathbf{R}_{t} \frac{\mathbf{d}_{\text {diff }}}{\left\|\mathbf{d}_{\text {diff }}\right\|}\left\|\mathbf{a}_{\text {trans }}\right\|
\end{gathered}
$$

where $\mathbf{d}_{\text {diff }}$ is the vector from the COM of $\mathbf{A}$ to $\mathbf{A}^{\prime}$ and $\mathbf{R}_{t}$ is a rotation matrix, rotating $\alpha=\left[-\operatorname{sign}\left(\theta_{r}\right)\right.$ $\left.\left(\left|\theta_{r}\right| / 2+\pi / 2\right)\right]$ rad about $\hat{\mathbf{r}}(\mathbf{F i g} . \mathbf{1 B})$.

\section{Constructing the Ideal $C_{n}$ Symmetric Pose that is Closest to a Non-Ideal Docking Pose or X-Ray Crystallographically Solved Structure}

No poses obtained from docking or experimentally resolved structures are in perfect $\mathrm{C}_{\mathrm{n}}$ symmetry (non-ideal). Our goal here is to obtain an ideal $\mathrm{C}_{\mathrm{n}}$ symmetric pose that is closest to the non-ideal pose. The deviation of the non-ideal pose from the ideal pose is used to determine if the non-ideal pose is $C_{n}$ symmetric (see $C_{n}$ Symmetry RMSD in Supplementary Methods).

To construct the ideal $\mathrm{C}_{\mathrm{n}}$ symmetric pose $\left(\mathrm{A}^{*}\right)$ from a non-ideal pose, the angle of rotation and translation vector are computed as follows

$$
\mathbf{d}_{\text {diff }}^{*}=\mathbf{d}_{\text {diff }}-\left(\mathbf{d}_{\text {diff }}^{T} \hat{\mathbf{r}}\right) \hat{\mathbf{r}}
$$

where $n=\left[\left|2 \pi / \theta_{r}\right|\right.$ rounded to the nearest integer] (such that $\theta_{n}=2 \pi / n$ ) and $\mathbf{d}_{\text {diff }}^{*}$ is the vector component of $\mathbf{d}_{\text {diff }}$ that is orthogonal to $\hat{\mathbf{r}}$. Note that, $\left|\mathbf{d}_{\text {diff }}^{T} \hat{\mathbf{r}}\right|$ is the shift along the AOS (see Supplementary Methods). 
Then, $\mathbf{a}_{\text {trans }}$ is computed with $\mathbf{d}_{\text {diff }}^{*}$ and $\theta_{n}$ (see Equation (4) and (5)). To obtain the A** pose, $\mathbf{A}_{0}$, $\mathbf{a}_{\text {trans }}$ and $\mathbf{R}^{*}$ are substituted into Equation (1), where the rotation matrix $\mathbf{R}^{*}$ rotates about $\hat{\mathbf{r}}$ by $\theta_{n}$.

\section{Data Sets}

\section{Non-redundant X-ray structures of $\alpha$-helical HoTPs}

The PDB IDs of $\alpha$-helical transmembrane proteins were taken from the mpstruct database (http://blanco.biomol.uci.edu/mpstruc/) and filtered with RCSB PDB's advanced filter (Rose et al., 2017) to remove redundant proteins and select for high-resolution X-ray structures (see Supplementary Methods). The structures were manually verified to be transmembrane and homo-oligomeric proteins resulting in the final data set containing 129 PDB IDs.

\section{SmFRET and structural data used for validation}

The Syt1-SNARE smFRET data are obtained from Choi et al. (Choi et al., 2010) and filtered such that each residue pair's transfer efficiency histogram has a single peak (Supplementary Fig. S1, Table S1 and S2). Syt1-SNARE's reference structure is PDB ID: 5CCG (Zhou et al., 2015) but the C2A and C2B domains of Syt1 are from PDB ID: 2R83 (Huang et al., 2013) which were used to fix the missing residue GLU130 (see Supplementary Methods). For MscL, the smFRET data (Supplementary Fig. S2 and Table S3) for the closed state E. coli MscL are from Wang et al. (Wang et al., 2014) while the reference structure is PDB ID: 2OAR (Steinbacher et al., 2007). The smFRET data (Supplementary Table S4) for the $C t \mathrm{H}^{+}$-PPase system are from Huang et al. (Huang et al., 2013) and the reference structure is predicted by homology modeling using SwissModel (Biasini et al., 2014) with the template structure from the $\mathrm{VrH}^{+}$-PPase system sharing a sequence identity of 45.1\% (PDB ID: 4A01 (Lin et al., 2012); the smFRET data for $V r \mathrm{H}^{+}$-PPase are mapped from $\mathrm{CtH}^{+}$-PPase to $\mathrm{VrH}^{+}$-PPase based on the pairwise sequence alignment (Supplementary Fig. S3). Further details can be found in the Supplementary Methods.

\section{Implementation of DR-SIP}

The DR-SIP package is implemented in Python 2.7 (McKinney, 2010; Gowers et al., 2016; Lam et al., 2015; Cock et al., 2009; Millman and Aivazis, 2011) and contains four modules: drsip, drsipcommon, zdock-parser and docking-eval. The drsip module implements the docking protocols, filters and a command-line-interface (CLI) for users to run the DR-SIP docking protocols. When the docking results are derived from ZDOCK, the zdock-parser module is available to parse the ZDOCK output file, generate the docking poses and write the coordinates out to PDB files. On the other hand, the docking-eval module implements the CAPRI criteria used to evaluate the quality of docking poses. Lastly, drsip-common contains functions that are commonly used by the other modules.

Regular users can perform standard DR-SIP docking protocols with the CLI while more advanced users can import the specific modules and functions to perform their own customized docking protocol.

The source code and documentation on how to use the DR-SIP package are provided at https://github.com/capslockwizard/drsip. The packages are also distributed through the PyPi and Anaconda Cloud repositories. 


\section{RESULTS}

\section{Statistical Analysis of 115 Structurally Solved $\alpha$-Helical HoTPs}

To observe how common $C_{n}$ symmetric structures are and how well the properties of $C_{n}$ symmetry holds for $\alpha$-helical HoTPs, 152 non-redundant structures containing HoTPs were taken from the mpstruct database (http://blanco.biomol.uci.edu/mpstruc/), see Supplementary Table file. Out of the 152 structures, 23 of them are monomers or do not contain a transmembrane domain. The remaining 129 structures (64\% are observed within asymmetric units) contain 118 structures (includes two dihedral symmetric structures, see Supplementary Methods) with parallel orientation (N-termini of constituent monomers face the same side of the membrane) and 11 antiparallel structures (see Supplementary Results). The 118 parallel structures which constitute $91.5 \%$ of the 129 HoTPs were used for subsequent statistical analyses. The anti-parallel structures are not included because most have not been verified as their functional forms through supporting experiments (see Supplementary Results).

The distribution of the sizes of the 118 parallel HoTPs (Fig. 3A) show that most are dimers (47 HoTPs), trimers (28 HoTPs) and tetramers (26 HoTPs), for a total of 101 HoTPs $(85.6 \%)$ out of the 118 HoTPs.

We further identified 115 of the 118 parallel HoTPs to be $\mathrm{C}_{\mathrm{n}}$ symmetric (Fig. 3B-E, $\mathrm{C}_{\mathrm{n}}$ symmetry RMSD <2 $\AA$ ) concluding that most of the HoTPs $(89.1 \%$ or $115 / 129)$ are $C_{n}$ symmetric and parallel in orientation. 
A

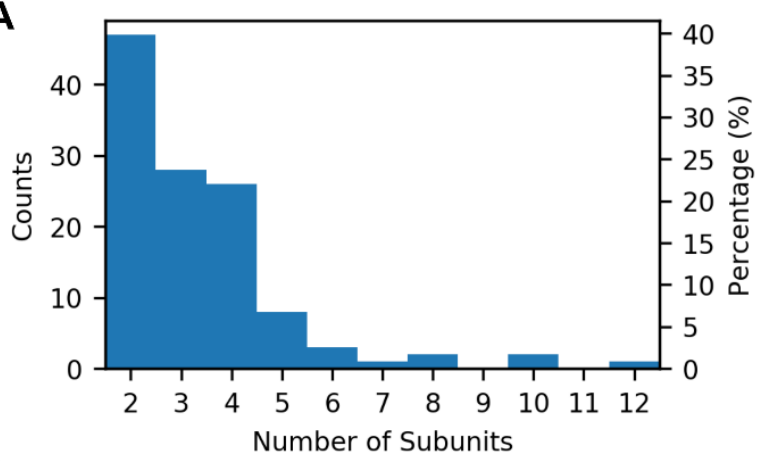

C

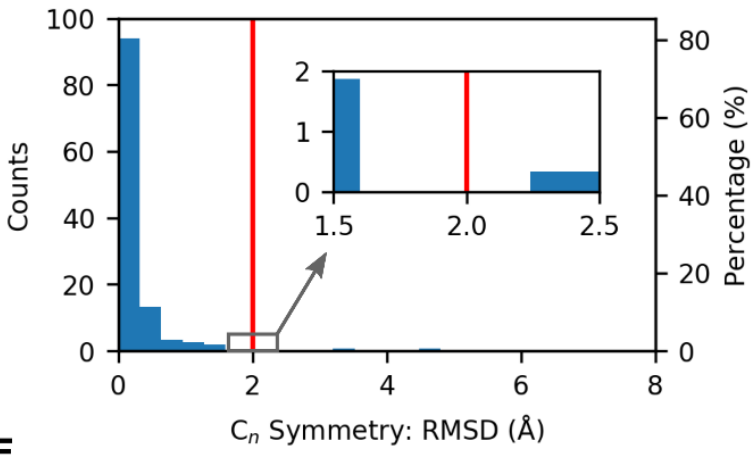

E

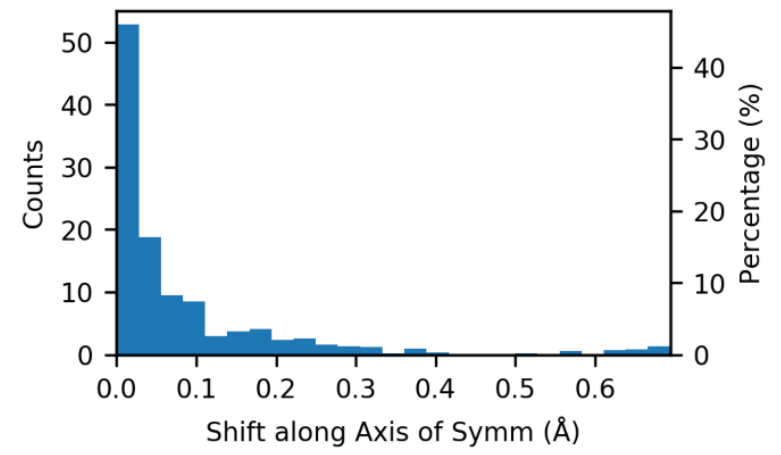

B

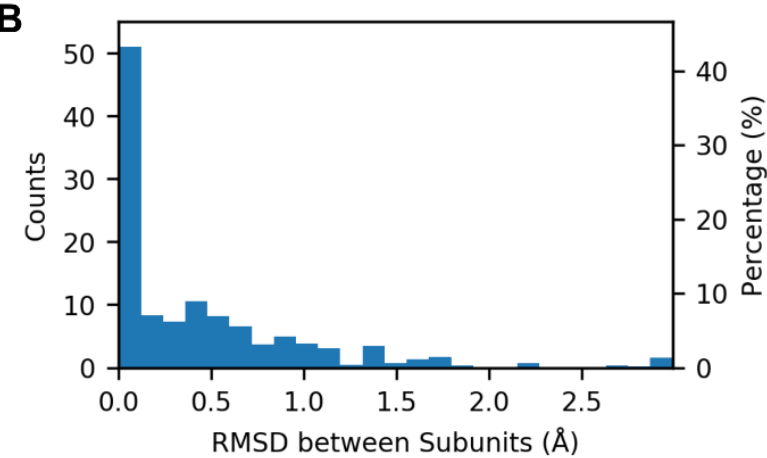

D

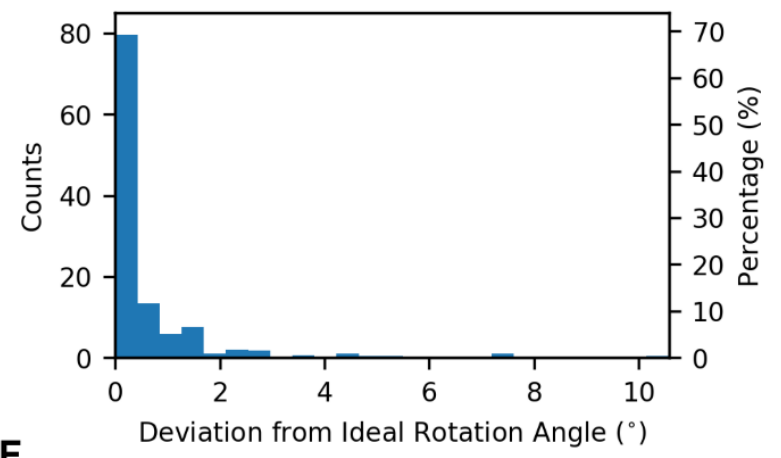

$\mathbf{F}$

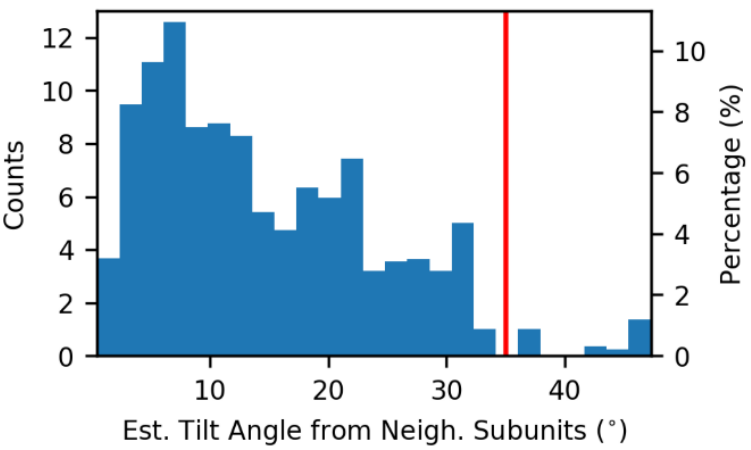

Fig. 3. Statistical distributions of the 118 HoTPs with parallel orientation. The left axis shows the counts of each bin while their percentages (out of 115 or 118 structures) are shown on the right axis. (A) Distribution of oligomeric states of the HoTPs reveals 85.6\% (101/118) of the structures are dimers, trimers and tetramers. (B) Conformational differences between neighboring monomers measured by root-mean squared deviation (RMSD) of $\mathrm{C} \alpha$ atoms, shows that the conformational differences are small, all are $<3 \AA$ while $87.0 \%<1 \AA$. (C) The $C_{n}$ symmetry RMSD measures the translational and rotational differences for a pair of neighboring monomers caused by deviation from ideal $\mathrm{C}_{\mathrm{n}}$ symmetry (see Methods). The distribution includes the HoTPs within $8 \AA$ (except for PDB ID: $5 \mathrm{HK} 1$ ). The cutoff of $<2 \AA$ (see red line and inset) is used to identify $\mathrm{C}_{\mathrm{n}}$ symmetric HoTPs. 115 (97.5\%) out of the 118 structures are within this cutoff. The remaining three structures (Supplementary Results), PDB IDs: 2GIF, 2QTS and 5HK1, with the lowest RMSD between neighboring monomers of $2.5 \AA, 4.6 \AA, 21.1 \AA$, respectively, were classified as non- $\mathrm{C}_{\mathrm{n}}$ symmetric. (D and E) The deviation from ideal $\mathrm{C}_{\mathrm{n}}$ symmetry for the remaining 115 structures can be decomposed into rotational differences and translational differences which are measured by the (D) deviation from ideal rotation angle and (E) shift along (parallel to) the AOS, respectively. The shift here refers to the amount of the translational difference between the two monomer's COM 
(d $\mathbf{d}_{\text {diff }}$, Fig. 1B) that are parallel to the AOS. (F) The tilt angle distribution shows the acute angle between the direction that the monomers span the membrane and the membrane normal (AOS). $97.4 \%$ of the $115 \mathrm{C}_{\mathrm{n}}$ symmetric structures have tilt angles $<35^{\circ}$ (red line) which is the cutoff employed in the SIP filter. (B-F) are calculated using all neighboring pairs of monomers in each HoTP. Each pair is weighted to contribute $1 / n$ to the counts except for homo-dimers where the weight is one. 


\section{Decomposition of the Deviation from Ideal $C_{n}$ Symmetry}

There are two potential contributors to the deviation from ideal $C_{n}$ symmetry. The first is the conformational difference between monomers in the complex. The other is the translational and rotational differences between a docking pose and its closest ideal $C_{n}$ symmetric pose.

Our results show that conformational differences between monomers is not a factor preventing the formation of $\mathrm{C}_{\mathrm{n}}$ symmetry. All the monomers in the 118 parallel HoTPs have small structural differences (Fig. 3B) - most ( 90\%) are less than $1 \AA$, while all are less than $3 \AA$.

On the other hand, the $\mathrm{C}_{\mathrm{n}}$ symmetry RMSD (Fig. 3C) measures the translational and rotational differences between the ideal $C_{n}$ symmetric pose and the pose from the X-ray structure for all unique pairs of neighboring monomers. When the $\mathrm{C}_{\mathrm{n}}$ symmetry RMSD is $<2.0 \AA$ (Fig. 3C, red line and inset), the pose is classified as $\mathrm{C}_{\mathrm{n}}$ symmetric. This is the same cutoff used in the SIP filter. Among the 118 parallel HoTPs, 115 (97.5\%) were classified as $C_{n}$ symmetric.

The cutoff of $<2.0 \AA$ was chosen because it is the midpoint between $1.6 \AA$ and $2.4 \AA$ where there are no structures (Fig. 3C). There are only three HoTPs (Supplementary Fig. S4 and Table S5) that have $C_{n}$ symmetry RMSD $>2.4 \AA$ and upon further examination were found to be non- $C_{n}$ symmetric (see Supplementary Results for details on the three HoTPs). Figure 4 compares the $\mathrm{X}$-ray structures of the two HoTPs closest to the $2.0 \AA$ cutoff, one above it and the other below it, with their respective ideal $C_{n}$ symmetric complex. The ExbB/ExbD complex (Fig. 4A) which is below the cutoff does not deviate much (see next section) from the ideal $C_{n}$ symmetric complex while the multidrug efflux pump subunit AcrB (Fig. 4B) shows a visibly larger deviation (see next section).

\section{Decomposition of Deviations from Ideal $C_{n}$ Symmetry into Translation and Rotational Differences}

Deviations from ideal $\mathrm{C}_{\mathrm{n}}$ symmetry are decomposed into translational (shift along AOS, sAOS) and rotational (deviation from ideal rotation angle, devRot) differences. The statistics show that all $115 \mathrm{C}_{\mathrm{n}}$ symmetric HoTPs have $<11^{\circ}$ deviation from the ideal rotation angle ( $94 \%$ is $<2^{\circ}$, Fig. 1D) and $<0.7 \AA$ shift along AOS (95\% is $<0.3 \AA$, Fig. 1E).

All the three non- $\mathrm{C}_{\mathrm{n}}$ symmetric structures (Supplementary Table S5) have $\geq 0.7 \AA$ sAOS while two out of the three (acid-sensing ion channel and the trimeric AcrB [Fig. 4B]) have $\geq 11^{\circ} \operatorname{devRot}$ with the remaining one $>6.0^{\circ}$. For comparison, the ExbB/ExbD complex (Fig. 4A) has $0.5 \AA$ sAOS and $4.0^{\circ}$ devRot which are smaller than the three non- $\mathrm{C}_{\mathrm{n}}$ symmetric structures.

\section{Tilt Angles Distribution}

The distribution of tilt angles (Fig. 1F) show that all the 115 structures have tilt angles $<50^{\circ}$, while $90 \%$ of them is $\angle 30^{\circ}$; hence $30^{\circ}$ (red line in Fig. 1F) is used as the tilt angle cutoff for the SIP filter. 


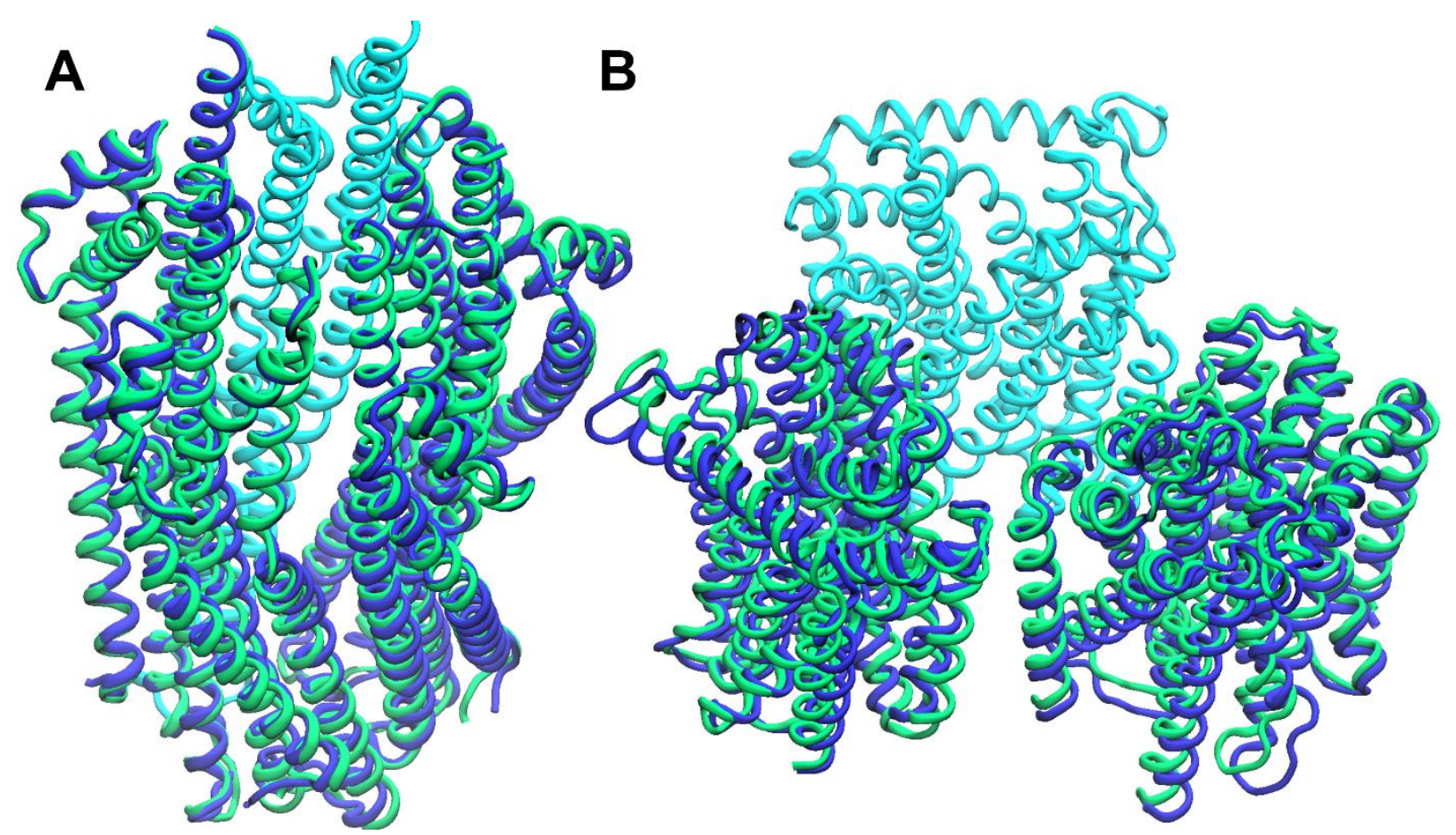

Fig. 4. Comparison of the X-ray structure (blue) and the ideal $\mathrm{C}_{\mathrm{n}}$ symmetric complex (green) for the two HoTPs closest to the $C_{n}$ symmetry RMSD cutoff of $2 \AA$. The complexes are superimposed at chain $A$ (cyan). (A) The protein that is closest to and below the cutoff is the ExbB/ExbD complex (PDB ID: 5SV0, $\mathrm{C}_{n}$ symmetry RMSD: $1.6 \AA$ ). (B) The protein that is closest to, but above the cutoff is the multidrug efflux pump subunit AcrB (PDB ID: 2GIF, RMSD: $2.5 \AA$ ). There are more visible differences when the RMSD is above the chosen cutoff of $2 \AA$. 


\section{SIP Filter Performance Without Distance Restraints}

Experimentally measured distances of interacting monomers in a HoTP complex are not commonly available. Even without distance restraints (DR), SIP alone can predict a near-native pose for every $\sim 3 / 4$ HoTPs (73\%, Table 1).

The 115 HoTP poses from the dataset were docked with ZDOCK and filtered by SIP. SIP increases the percentage of HoTPs that can be found within the top-3, 5, 10 and 20 ranking results (Table 1). The largest increase comes from improving the number of HoTPs with near-native pose(s) within the top-20 (DR-SIP: 73\% of 115 HoTPs, ZDOCK: 47.8\%) and top-10 (DR-SIP: 64.3\%, ZDOCK: $45.2 \%$ ) results.

Since the dataset is dominated by dimers (40.9\% of HoTP, Fig. 3A), it is possible that SIP is just good at predicting dimers. By excluding dimers from the statistics, we show that SIP performs just as well (top-20: $67.6 \%$, top-10: 57.4\%) for higher order oligomers, while the performance of ZDOCK drops to 32 and 28\%, respectively (Table 1).

Near-native docking poses are identified by comparing each docking pose to their respective reference X-ray structure by a modified CAPRI criteria (Méndez et al., 2003) for HoTPs which assigns each pose into one of four ranks: High, Medium, Acceptable and Incorrect, respectively (see Supplementary Methods and Table S6). Docking poses that are not "Incorrect" ( $[<10 \%$ native contacts] or [>4.0 iRMSD and >10.0 IRMSD] or [have a different order of symmetry with the reference structure]) are considered as near-native poses. 
Table 1. Percentage of 115 HoTPs Whose Near-Native Pose Can Be Recovered by SIP or ZDOCK Without Distance Restraints.

\begin{tabular}{|c|c|c|c|c|}
\hline & Top-20 (\%) & Top-10 (\%) & Top-5 (\%) & Top-3 (\%) \\
\hline ZDOCK $^{\text {a,c }}$ & 47.8 & 45.2 & 40.9 & 39.1 \\
\hline DR-SIP (Ex. DR) $^{\mathbf{b , c}}$ & 73.0 & 64.3 & 52.2 & 42.6 \\
\hline ZDOCK Rank (Ex. Dimers) $^{\mathbf{d}}$ & 32.4 & 27.9 & 23.5 & 23.5 \\
\hline DR-SIP (Ex. DR) Rank (Ex. Dimers) $^{\mathbf{d}}$ & 67.6 & 57.4 & 48.5 & 39.7 \\
\hline
\end{tabular}

All 115 HoTPs were docked with ZDOCK. Shows the percentage of the HoTPs where at least one near-native pose can be found within the top-3, 5, 10 and 20 results. Near-native poses are identified using the modified CAPRI criteria for HoTPs (see Supplementary Methods and Table S6).

${ }^{a}$ For each HoTP, the original ZDOCK rank of the unfiltered 54,000 docking poses.

${ }^{b}$ For each HoTP, the SIP filter $\left(C_{n}\right.$ symmetry $>2 \AA$, tilt angle $\left.>35^{\circ}\right)$ and clustering are applied on the 54,000 docking poses. The clusters and their respective representative poses are ranked by size in descending order. See Methods and Fig. 2.

${ }^{\mathrm{c}}$ Percentage of the 115 HoTPs.

${ }^{\mathrm{d}}$ Percentage of the remaining 68 HoTPs after excluding all 47 dimers. 


\section{Performance of DR-SIP with Distance Restraints: Near-Native Poses Within the Top-2 Ranking Poses}

The performance of DR-SIP when distance restraints are available is evaluated by applying the docking protocols (Fig. 2) to one soluble protein system (Syt1-SNARE) and three HoTP systems (MscL, $V r \mathrm{H}^{+}$-PPase and $C t \mathrm{H}^{+}$-PPase). The results are compared to DR-SIP without distance restraints and the original ZDOCK ranking (Table 2).

A near-native pose of $\mathrm{Vr} \mathrm{H}^{+}$-PPase and $\mathrm{Ct}_{\mathrm{H}}^{+}$-PPase can be found within the top-20 ZDOCK ranked poses but not for Syt1-SNARE and MscL. Consistent with the results of the previous section, applying SIP without the DR filter on MscL increases the chance to find a near-native pose within the top-10. Indeed, one is found at the $7^{\text {th }}$-ranked pose. The ranking is further improved to $2^{\text {nd }}($ Fig. $\mathbf{5 A}, \mathbf{B}$ ) when the DR filter is added. While for the soluble system, Syt1-SNARE (a hetero-oligomer where $C_{n}$ symmetry does not apply), applying only the DR filter, the top-ranked pose (Fig. 5B) is the near-native pose.

DR-SIP performs equivalently for systems that are ranked $1^{\text {st }}$ by ZDOCK such as $V r \mathrm{H}^{+}$-PPase and $C t \mathrm{H}^{+}$-PPase. We want to emphasize that DR-SIP does not rely on the ranking of ZDOCK, or the ranking of any other software that generates the initial pool of docking poses. For "tougher" systems, DR-SIP enriches the near-native poses such that they can be found within the top-2 results (Table 2 and Fig. 5). 
Table 2. Improvement of the Ranking of the Top-Ranked Near-Native Docking Poses with DR-SIP.

\begin{tabular}{|c|c|c|c|c|}
\hline & Syt1-SNARE & MscL & $\boldsymbol{C t}^{+} \mathbf{H}^{+}$-PPase & $\boldsymbol{V r H}^{+}$-PPase \\
\hline ZDOCK $^{\mathbf{a}}$ & 62 & 2231 & 1 & 1 \\
\hline SIP only (Ex. DR) $^{\mathbf{b}}$ & - & 7 & 1 & 1 \\
\hline DR-SIP & 1 & 2 & 1 & 1 \\
\hline
\end{tabular}

Shows the top ranking near-native pose for four systems: Syt1-SNARE, MscL, $C t \mathrm{H}^{+}-\mathrm{PPase}$ and $V r \mathrm{H}^{+}-\mathrm{PPase}$.

${ }^{a}$ The original ZDOCK rank of the 54,000 unfiltered poses for all four systems.

${ }^{b}$ DR-SIP ranking obtained by applying only the SIP filter $\left(C_{n}\right.$ symmetry $>2 \AA$, tilt angle $\left.>35^{\circ}\right)$, just like in Table 1 .

${ }^{c}$ DR-SIP ranking obtained by applying both the DR filter $\left(\leq 0.3\right.$ correlation) and SIP filter $\left(\mathrm{C}_{\mathrm{n}}\right.$ symmetry $>2 \AA$, tilt angle $>35^{\circ}$ ), see Methods and Fig. 2. 

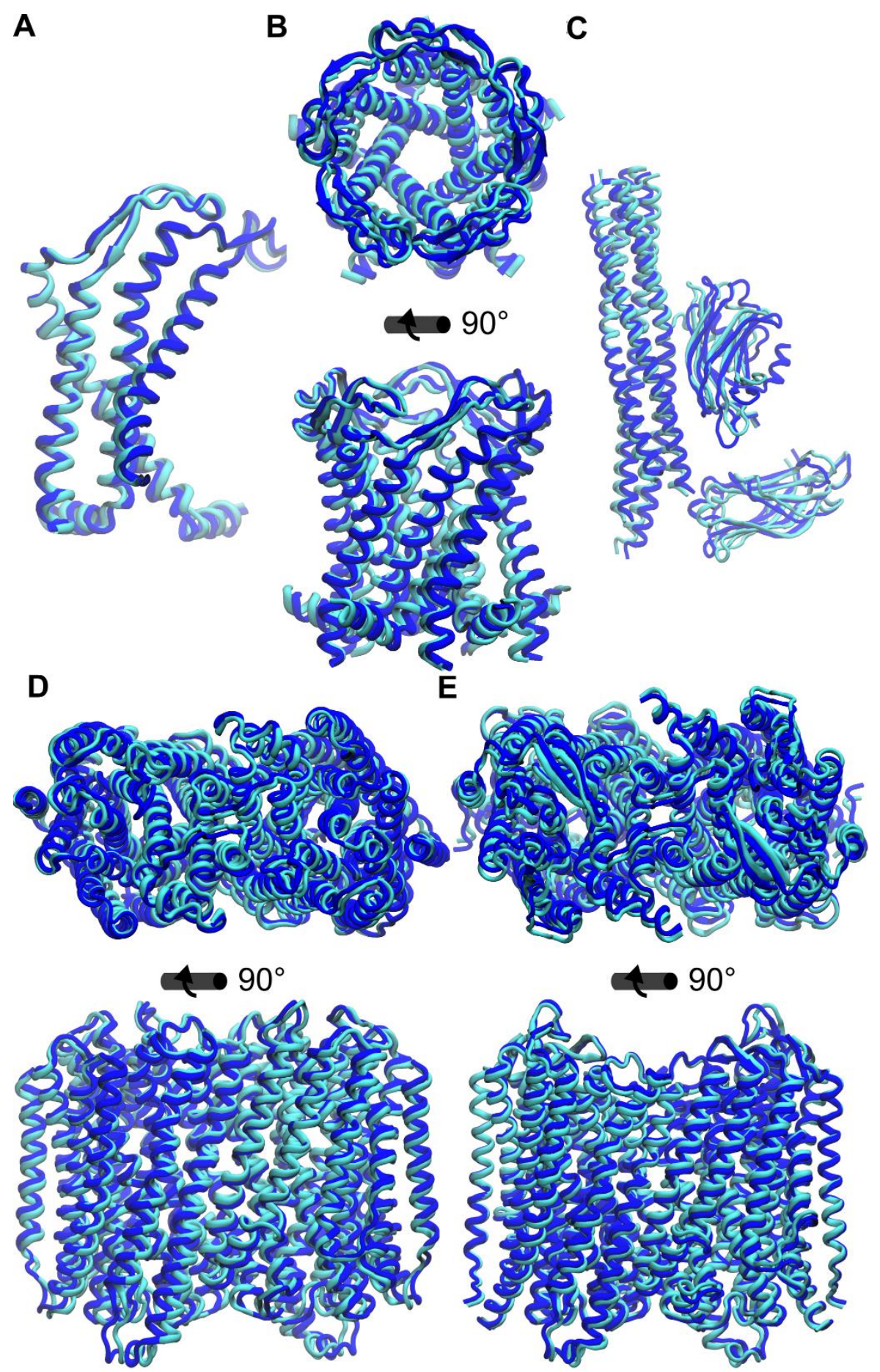

Fig. 5. The top-ranked near-native docking pose (cyan) predicted by DR-SIP superimposed onto the reference structure (blue) for all four systems with distance restraints. (A-B) MscL: $2^{\text {nd }}$ ranked pose with the reference structure from PDB ID: 2OAR. (A) The docking pose (dimer) superimposed onto chain A and B of the reference structure. (B) The predicted pentamer complex with the reference structure. $(\mathbf{C})$ Syt1-SNARE complex: the $1^{\text {st }}$ ranked DR-SIP pose with the reference structure PDB ID: 5CCG. (D) $C t \mathrm{H}^{+}-\mathrm{PPase}: 1^{\text {st }}$ ranked pose with the homology modeled structure based on the template PDB ID: 4A01. (E) $V r \mathrm{H}^{+}$-PPase: $1^{\text {st }}$ ranked pose with the reference structure PDB ID: 4A01. 
Table 3. Contribution of the DR-SIP Filters to Enriching the Near-Native Poses Generated by ZDOCK.

\begin{tabular}{|c|c|c|c|c|c|c|c|}
\hline & \multirow{2}{*}{$\%$ Native $^{\mathrm{c}}$} & \multirow{2}{*}{ Enrichment $^{\mathrm{d}}$} & \multicolumn{3}{|c|}{ Native Poses } & \multirow{2}{*}{ Incorrect } & \multirow{2}{*}{ Total } \\
\hline & & & High & Medium & Acceptable & & \\
\hline \multicolumn{8}{|c|}{ Syt1-SNARE } \\
\hline Unfiltered & 0.25 & 1.00 & 3 & 23 & 108 & 53866 & 54000 \\
\hline All Filters ${ }^{\mathrm{a}}$ & 2.70 & 10.89 & 3 & 23 & 108 & 4824 & 4958 \\
\hline Filtered $^{\mathrm{a}}$ \& Clustered & 1.65 & 6.64 & 0 & 1 & 6 & 418 & 425 \\
\hline Top 2 with DR & 50.00 & 201.49 & 0 & 0 & 1 & 1 & 2 \\
\hline \multicolumn{8}{|c|}{ MscL } \\
\hline Unfiltered & 0.04 & 1.00 & 4 & 11 & 5 & 53980 & 54000 \\
\hline All Filters $^{b}$ & 9.73 & 243.25 & 4 & 7 & 0 & 102 & 113 \\
\hline Ex. $C_{n}$ Symm. RMSD & 0.56 & 14.00 & 4 & 8 & 1 & 2294 & 2307 \\
\hline Ex. Tilt Angle & 1.38 & 34.50 & 4 & 8 & 1 & 931 & 944 \\
\hline Ex. DR Filter & 2.35 & 58.75 & 4 & 7 & 0 & 457 & 468 \\
\hline Ex. DR Filter \& Clustered & 4.17 & 104.25 & 0 & 1 & 0 & 23 & 24 \\
\hline Filtered $^{\mathrm{b}}$ \& Clustered & 11.11 & 277.75 & 0 & 1 & 0 & 8 & 9 \\
\hline Top 7 (Ex. DR) & 14.29 & 357.25 & 0 & 1 & 0 & 6 & 7 \\
\hline Top 2 (Ex. DR) & 0.00 & 0.00 & 0 & 0 & 0 & 2 & 2 \\
\hline Top 7 with DR & 14.29 & 357.25 & 0 & 1 & 0 & 6 & 7 \\
\hline Top 2 with DR & 50.00 & 1250.00 & 0 & 1 & 0 & 1 & 2 \\
\hline \multicolumn{8}{|c|}{ Ct $\mathrm{H}^{+}$-PPase } \\
\hline Unfiltered & 0.14 & 1.00 & 0 & 29 & 45 & 53926 & 54000 \\
\hline All Filters $^{b}$ & 26.15 & 186.79 & 0 & 19 & 15 & 96 & 130 \\
\hline Ex. Cn $_{n}$ Symm. RMSD & 2.12 & 15.14 & 0 & 29 & 45 & 3417 & 3491 \\
\hline Ex. Tilt Angle & 9.69 & 69.21 & 0 & 19 & 15 & 317 & 351 \\
\hline Ex. DR Filter & 10.56 & 75.43 & 0 & 19 & 15 & 288 & 322 \\
\hline Ex. DR Filter \& Clustered & 3.57 & 25.50 & 0 & 1 & 0 & 27 & 28 \\
\hline Filtered $^{\mathrm{b}} \&$ Clustered & 12.50 & 89.29 & 0 & 1 & 0 & 7 & 8 \\
\hline Top 7 (Ex. DR) & 14.29 & 102.07 & 0 & 1 & 0 & 6 & 7 \\
\hline Top 2 (Ex. DR) & 50.00 & 357.14 & 0 & 1 & 0 & 1 & 2 \\
\hline Top 7 with DR & 14.29 & 102.07 & 0 & 1 & 0 & 6 & 7 \\
\hline Top 2 with DR & 50.00 & 357.14 & 0 & 1 & 0 & 1 & 2 \\
\hline \multicolumn{8}{|c|}{ VrH ${ }^{+}$-PPase } \\
\hline Unfiltered & 0.17 & 1.00 & 4 & 29 & 58 & 53909 & 54000 \\
\hline All Filters $^{\mathbf{b}}$ & 16.67 & 98.06 & 3 & 13 & 15 & 155 & 186 \\
\hline 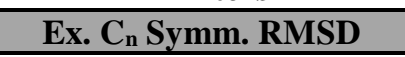 & 2.00 & 11.76 & 4 & 29 & 58 & 4451 & 4542 \\
\hline Ex. Tilt Angle & 7.49 & 44.06 & 3 & 13 & 15 & 383 & 414 \\
\hline Ex. DR Filter & 7.60 & 44.71 & 3 & 13 & 15 & 377 & 408 \\
\hline Ex. DR Filter \& Clustered & 2.33 & 13.71 & 0 & 1 & 0 & 42 & 43 \\
\hline Filtered $^{\mathrm{b}}$ \& Clustered & 6.25 & 36.76 & 0 & 1 & 0 & 15 & 16 \\
\hline Top 7 (Ex. DR) & 14.29 & 84.06 & 0 & 1 & 0 & 6 & 7 \\
\hline Top 2 (Ex. DR) & 50.00 & 294.12 & 0 & 1 & 0 & 1 & 2 \\
\hline Top 7 with DR & 14.29 & 84.06 & 0 & 1 & 0 & 6 & 7 \\
\hline Top 2 with DR & 50.00 & 294.12 & 0 & 1 & 0 & 1 & 2 \\
\hline
\end{tabular}

The "Ex." here means that all the other filters/criteria were applied except the listed filter/criterion. The docking poses are ranked into one of the following: High, Medium, Acceptable or Incorrect, according to the modified CAPRI criteria (see Supplementary Methods and Table S6).

${ }^{a}$ Using only the DR filter ( $₫ 0.3$ correlation). 
bioRxiv preprint doi: https://doi.org/10.1101/500397; this version posted January 8,2019 . The copyright holder for this preprint (which was not certified by peer review) is the author/funder. All rights reserved. No reuse allowed without permission.

${ }^{\mathrm{b}}$ Using both the DR filter $\left(\leq 0.3\right.$ correlation) and SIP filter $\left(\mathrm{C}_{\mathrm{n}}\right.$ symmetry $>2 \AA$, tilt angle $\left.>35^{\circ}\right)$.

c $\%$ Native $=($ High + Medium + Acceptable $)$ Poses $/$ Total Poses $* 100$.

${ }^{\mathrm{d}}$ Enrichment $=(\%$ Native $) /(\%$ Native when unfiltered $)$. For example, MscL's "Top 2 with DR" enrichment of 1250.0 is obtained by dividing the $50 \%$ near-native poses with the $\sim 0.04 \%$ near-native poses in the unfiltered data. 


\section{Contribution of the Filters and Clustering to the Enrichment of the Near-Native Poses}

Among the 54,000 docking poses generated by ZDOCK for each of the four systems, $<0.3 \%$ (Table 3) of the poses are near-native poses. The docking protocols enriched the near-native poses up to 1250-fold such that at least one can be found within the top-2 ranking results (Table 2 and 3).

The contribution of the filters is measured by excluding one of the four filters/criteria and comparing the decrease in the enrichment of near-native poses relative to the enrichment when using all filters/criteria (Table 3). The larger the drop in the enrichment, the larger the contribution of that filter.

The $\mathrm{C}_{\mathrm{n}}$ symmetry criterion has the largest contribution to the enrichment resulting in a drop of $\sim 90 \%$ (in Table 3, [Ex. C Symm. RMSD/All Filters - 1] x 100\%) when excluded. On the other hand, the tilt angle criterion and relative distance filters are of equal importance, reducing the enrichment by $\sim 55-85 \%$ when excluded.

After applying all the filters, the near-native poses are significantly enriched (up to 243-fold) and make up 10\%-25\% of the remaining poses. The near-native poses are further enriched by clustering of the poses (see METHODS) such that the near-native pose can be found within the top-2 results.

A near-native pose of MscL cannot be found in the top-2 results without the DR filter. MscL starts off with 3 to 4 -fold fewer near-native poses ( $0.04 \%$ out of 54,000 poses) compared to the other two HoTPs $(\sim 0.16 \%)$. Without the DR filter, the SIP filter can only enrich the near-native poses to $2.35 \%$ of poses. This is enough to obtain a near-native pose at the $7^{\text {th }}$-ranked pose (Table 2). A 4-fold enrichment by the DR filter pushes the final rank to $2^{\text {nd }}$ (Table 2).

For the soluble case, the Syt1-SNARE complex, the DR filter enriches the near-native poses by $\sim 11$-fold. Clustering and ranking the remaining poses results in a $1^{\text {st }}$-ranked near-native pose.

\section{Performance of DR-SIP with GRAMM-X}

To examine how DR-SIP performs with other docking software, DR-SIP is applied to the docking poses generated from GRAMM-X for all four systems. From the maximum 300 poses that could be obtained for each system, GRAMM-X results contain the near-native pose(s) for MscL, $V r \mathrm{H}^{+}-$ PPase and $\mathrm{Ct}^{+} \mathrm{H}^{+}$PPase but not for the Syt1-SNARE complex. Applying DR-SIP (excluding Syt1SNARE) enriches the near-native poses to $\sim 33-50 \%$ of remaining poses from the unfiltered 0.3 $2.7 \%$ (Table 4), performing similarly to the ZDOCK results (Table 3), which shows the generality of DR-SIP's performance as different docking packages are used. 
Table 4. GRAMM-X Docking Results Filtered by the DR-SIP Protocols.

\begin{tabular}{|c|c|c|c|}
\hline System & $\begin{array}{c}\text { \% Native Poses } \\
\text { [Before Filter] }\end{array}$ & $\begin{array}{c}\text { \% Native Poses } \\
\text { [After Filter] }^{\mathbf{a}}\end{array}$ & Enrichment $^{\mathbf{b}}$ \\
\hline MscL & $2.7(8 / 300)$ & $33.3(2 / 6)$ & 12.3 \\
\hline $\boldsymbol{C t}$-PPase & $0.3(1 / 300)$ & $50.0(1 / 2)$ & 166.7 \\
\hline $\boldsymbol{r}$-PPase & $0.3(1 / 300)$ & $33.3(1 / 3)$ & 111.0 \\
\hline Syt1-SNARE & $0.0(0 / 300)$ & $0.0(0 / 300)$ & - \\
\hline
\end{tabular}

GRAMM-X returns a maximum of 300 top docking poses. The parentheses show the number of (near-native poses/total poses). Near-native poses are identified by the modified CAPRI criteria (see Supplementary Methods and Table S6).

${ }^{a}$ Using both the DR filter $\left(\leq 0.3\right.$ correlation) and SIP filter $\left(C_{n}\right.$ symmetry $>2 \AA$, tilt angle $\left.>35^{\circ}\right)$. No clustering was performed because there are too few poses after filtering.

${ }^{\mathrm{b}}$ Enrichment $=\%$ Native Poses [After Filter] $/ \%$ Native Poses [Before Filter]. 


\section{DISCUSSIONS}

The distribution of the " $n$ " of $\mathrm{C}_{n}$ symmetric HoTPs (Fig. S5A and Table S7) is not uniformly distributed, with dimers and trimers dominating the population. Such a distribution could occur from a natural outcome of chemical/geometric complementarity of the constituent monomers (physical reason) or meeting the functional needs of life (biological reason).

To see whether a purely physical model can reproduce the observed distribution of " $n$ ", we perform molecular docking with ZDOCK on three HoTP systems (MscL, $\mathrm{VrH} \mathrm{H}^{+}$-PPase and $\mathrm{CtH}^{+}$-PPase) and use the $\mathrm{C}_{\mathrm{n}}$ symmetry criterion to select only symmetric poses. ZDOCK, as an off-lattice model (Huang, 2014), uniformly samples the translational and rotational degrees of freedom and returns the top-54,000 poses with the best interaction scores between two identical monomers.

Our results show that the proportion of $\mathrm{C}_{2}$ 's is over-represented, $\sim 73.8 \%$ of total, (Fig. S5A, S5C and Table S7) relative to the observed $40.9 \%$ in the HoTP dataset. Not unique to HoTPs, $\mathrm{C}_{\mathrm{n}}$ symmetric soluble proteins (Pentameric Capsid Protein [PDB ID: 4DMI] and GH3 BetaGlucosidase [PDB ID: 5XXL]), selected for having a similar size as MscL and $V r \mathrm{H}^{+}-\mathrm{PPase} / C t \mathrm{H}^{+}-$ PPase, respectively (see Supporting Methods for details), also show $\mathrm{C}_{2}$ 's are overpopulated $(\sim 69.5 \%)$.

The discrepancy between our results and the observed distribution could be due the lack of consideration given to the constraints imposed on HoTPs as they span the membrane bilayer. One such constraint is the need for transmembrane regions of the monomers in HoTPs to be embedded in the membrane. When the transmembrane regions are longer than the thickness of the membrane, the monomers must undergo a tilt to fully embed these regions (Park and Opella, 2005; Kim and Im, 2010; de Jesus and Allen, 2013). Our statistics (Fig. 3F) show that a relatively small tilt angle $\left(<35^{\circ}\right.$; compared to the mean angle of $57.3^{\circ}$ ( $\mathrm{Li}$ et al., 2014) for two random lines in space) is preferred, which implies that most of the transmembrane regions are not too long. While, large tilt angles imply that the transmembrane region is longer, and this might be disadvantageous to the formation of sufficient binding interface in $\mathrm{C}_{2}$ complexes and/or cost-effectiveness of protein synthesis.

After applying the tilt angle criterion $\left(<35^{\circ}\right)$ to the $\mathrm{C}_{\mathrm{n}}$ symmetric ZDOCK poses, we observe a distribution that is similar $\left(\mathrm{C}_{2}\right.$ make up $\left.~ 53.6 \%\right)$ to the HoTP dataset (Fig. S5B, S5D and Table S7). Therefore, the tilt angle constraint is one of the major determinants of the observed distribution. However, there is no straightforward physical explanation of why tilt angles are predominantly populated over $7^{\circ}$ and $20^{\circ}$ (Fig. 3F). Therefore, we surmise that this "selection" is made for functional reasons such as proper functional dynamics or genetic reasons to provide evolutionary advantages.

The monomers in a $\mathrm{C}_{2}$ complex share the same binding interface but the monomers in the other $\mathrm{C}_{\mathrm{n}}$ 's interact at different interfaces. Therefore, any interaction between residues at a $\mathrm{C}_{2}$ interface comes in pairs (Monod et al., 1965). The interaction energy of good/bad interactions are doubled resulting in a larger variance of the interaction energies for $\mathrm{C}_{2}$ 's (André et al., 2008). Thus, we're are more likely to find $\mathrm{C}_{2}$ 's with very favorable interaction energies compared to other types of dimers (André et al., 2008). Since molecular docking returns poses with the most favorable interactions, we expect and indeed observe that $\mathrm{C}_{2}$ 's are enriched compared to other $\mathrm{C}_{\mathrm{n}}$ 's (Fig. 
S5A and Table S7). Thus, physical interactions can explain why $\mathrm{C}_{2}$ HoTPs are so common. However, only a subset of these $\mathrm{C}_{2}$ 's within the range of allowable tilt angles were chosen by evolution for functional purposes.

Taken together, DR-SIP incorporates geometric restraints such as $\mathrm{C}_{\mathrm{n}}$ symmetry and tilt angle for HoTPs while leveraging experimentally determined distance restraints providing a reliable opportunity to computationally assemble near-native quaternary structures of hetero-/homooligomers. 


\section{Acknowledgments}

Vast computational resources for this project are provided by National Center for Highperformance Computing (NCHC) of National Applied Research Laboratories (NARLabs), Taiwan. J.C. acknowledges financial support from Taiwan International Graduate Program (TIGP), Academia Sinica, Taiwan, as well as Dr. Tzyy-Jen Chiou's support (AS 103-TP-B11). This work is supported by the Ministry of Science and Technology, Taiwan (103-2627-M-007-001 and 1042113-M-007-019 to L.-W. Y.). 


\section{References}

Alber,F. et al. (2008) Integrating diverse data for structure determination of macromolecular assemblies. Annu. Rev. Biochem., 77, 443-477.

Alford,R.F. et al. (2015) An integrated framework advancing membrane protein modeling and design. PLoS Comput. Biol., 11, e1004398.

André,I. et al. (2008) Emergence of symmetry in homooligomeric biological assemblies. Proc. Natl. Acad. Sci. U. S. A., 105, 16148-16152.

Bertoni,M. et al. (2017) Modeling protein quaternary structure of homo- and hetero-oligomers beyond binary interactions by homology. Sci. Rep., 7, 1-15.

Biasini,M. et al. (2014) SWISS-MODEL: Modelling protein tertiary and quaternary structure using evolutionary information. Nucleic Acids Res., 42, W252-8.

Brooijmans,N. and Kuntz,I.D. (2003) Molecular recognition and docking algorithms. Annu. Rev. Biophys. Biomol. Struct., 32, 335-73.

Chang,C.-C. et al. (2016) Blocking the interaction between S100A9 and RAGE V domain using CHAPS molecule: A novel route to drug development against cell proliferation. Biochim. Biophys. Acta - Proteins Proteomics, 1864, 1558-1569.

Chaudhury,S. and Gray,J.J. (2008) Conformer selection and induced fit in flexible backbone protein-protein docking using computational and NMR ensembles. J. Mol. Biol., 381, 106887.

Choi,U.B. et al. (2010) Single-molecule FRET-derived model of the synaptotagmin 1-SNARE fusion complex. Nat. Struct. Mol. Biol., 17, 318-24.

Cock,P.J.A. et al. (2009) Biopython: Freely available Python tools for computational molecular biology and bioinformatics. Bioinformatics, 25, 1422-3.

Dimura,M. et al. (2016) Quantitative FRET studies and integrative modeling unravel the structure and dynamics of biomolecular systems. Curr. Opin. Struct. Biol., 40, 163-185.

Fagerberg,L. et al. (2010) Prediction of the human membrane proteome. Proteomics, 10, 11411149.

Gowers,R. et al. (2016) MDAnalysis: A Python package for the rapid analysis of molecular dynamics simulations. In, Benthall,S. and Rostrup,S. (eds), Proceedings of the 15th Python in Science Conference. Austin, TX, pp. 98-105.

Gupta,A.A. et al. (2013) Structural insights into the interaction of human S100B and basic fibroblast growth factor (FGF2): Effects on FGFR1 receptor signaling. Biochim. Biophys. Acta - Proteins Proteomics, 1834, 2606-2619.

Huang,S.Y. (2014) Search strategies and evaluation in protein-protein docking: Principles, advances and challenges. Drug Discov. Today, 19, 1081-1096.

Huang,Y.-T. et al. (2013) Squeezing at entrance of proton transport pathway in protontranslocating pyrophosphatase upon substrate binding. J. Biol. Chem., 288, 19312-20. 
Hurwitz,N. et al. (2016) Memdock: An $\alpha$-helical membrane protein docking algorithm. Bioinformatics, 32, 2444-50.

de Jesus,A.J. and Allen,T.W. (2013) The determinants of hydrophobic mismatch response for transmembrane helices. Biochim. Biophys. Acta, 1828, 851-863.

Jia,Y.-B. (2017) Rotation in the space. Com S 477/577 Notes, 1-11.

Kabsch,W. (1976) A solution for the best rotation to relate two sets of vectors. Acta Crystallogr. Sect. A, 32, 922-923.

Kalinin,S. et al. (2012) A toolkit and benchmark study for FRET-restrained high-precision structural modeling. Nat. Methods, 9, 1218-1227.

Khan,M.I. et al. (2018) S100B as an antagonist to block the interaction between S100A1 and the RAGE V domain. PLoS One, 13, e0190545.

Kim,T. and Im,W. (2010) Revisiting hydrophobic mismatch with free energy simulation studies of transmembrane helix tilt and rotation. Biophys. J., 99, 175-183.

Kozakov,D. et al. (2017) The ClusPro web server for protein-protein docking. Nat. Protoc., 12, 255-278.

Lam,S.K. et al. (2015) Numba. In, Proceedings of the Second Workshop on the LLVM Compiler Infrastructure in HPC - LLVM '15. ACM Press, New York, New York, USA, pp. 1-6.

Levy,E.D. et al. (2008) Assembly reflects evolution of protein complexes. Nature, 453, 12621265.

Li,H. et al. (2014) Molecular binding sites are located near the interface of intrinsic dynamics domains (IDDs). J. Chem. Inf. Model., 54, 2275-85.

Liang,B. and Tamm,L.K. (2016) NMR as a tool to investigate the structure, dynamics and function of membrane proteins. Nat. Struct. Mol. Biol., 23, 468-474.

Lin,S.-M. et al. (2012) Crystal structure of a membrane-embedded H+-translocating pyrophosphatase. Nature, 484, 399-403.

Manning,C.D. et al. (2008) Introduction to information retrieval 1st ed. Cowles,L. (ed) Cambridge University Press, New York, NY, USA.

McKinney,W. (2010) Data structures for statistical computing in Python. In, Proceedings of the 9th Python in Science Conference., pp. 51-56.

Méndez,R. et al. (2003) Assessment of blind predictions of protein-protein interactions: Current status of docking methods. Proteins Struct. Funct. Genet., 52, 51-67.

Millman,K.J. and Aivazis,M. (2011) Python for scientists and engineers. Comput. Sci. Eng., 13, 9-12.

Mitra,K. and Frank,J. (2006) Ribosome dynamics: Insights from atomic structure modeling into cryo-electron microscopy maps. Annu. Rev. Biophys. Biomol. Struct., 35, 299-317.

Monod,J. et al. (1965) On the nature of allosteric transitions: A plausible model. J. Mol. Biol., 12, 
$88-118$.

Moraes,I. et al. (2014) Membrane protein structure determination - The next generation. Biochim. Biophys. Acta - Biomembr., 1838, 78-87.

Muschielok,A. et al. (2008) A nano-positioning system for macromolecular structural analysis. Nat. Methods, 5, 965-971.

Park,S.H. and Opella,S.J. (2005) Tilt angle of a trans-membrane helix is determined by hydrophobic mismatch. J. Mol. Biol., 350, 310-318.

Pierce,B. et al. (2005) M-ZDOCK: A grid-based approach for Cn symmetric multimer docking. Bioinformatics, 21, 1472-1478.

Pierce,B.G. et al. (2011) Accelerating protein docking in ZDOCK using an advanced 3D convolution library. PLoS One, 6, e24657.

Rask-Andersen,M. et al. (2014) The druggable genome: Evaluation of drug targets in clinical trials suggests major shifts in molecular class and indication. Annu. Rev. Pharmacol. Toxicol., 54, 9-26.

Rose,P.W. et al. (2017) The RCSB protein data bank: Integrative view of protein, gene and 3D structural information. Nucleic Acids Res., 45, D271-D281.

Sasmal,D.K. et al. (2016) Single-molecule fluorescence resonance energy transfer in molecular biology. Nanoscale, 8, 19928-19944.

Schneidman-Duhovny,D. et al. (2005) PatchDock and SymmDock: Servers for rigid and symmetric docking. Nucleic Acids Res., 33, W363-W367.

Steinbacher,S. et al. (2007) Structures of the prokaryotic mechanosensitive channels MscL and MscS. In, Mechanosensitive Ion Channels, Part A., pp. 1-24.

Szilagyi,A. and Zhang,Y. (2014) Template-based structure modeling of protein-protein interactions. Curr. Opin. Struct. Biol., 24, 10-23.

Tovchigrechko,A. and Vakser,I.A. (2006) GRAMM-X public web server for protein-protein docking. Nucleic Acids Res., 34, W310-W314.

Viswanath,S. et al. (2015) Extension of a protein docking algorithm to membranes and applications to amyloid precursor protein dimerization. Proteins, 83, 2170-2185.

van der Walt,S. et al. (2011) The NumPy array: A structure for efficient numerical computation. Comput. Sci. Eng., 13, 22-30.

Wang,Y. et al. (2014) Single molecule FRET reveals pore size and opening mechanism of a mechano-sensitive ion channel. Elife, 2014, 1-21.

Yamagata,A. and Tainer,J.A. (2007) Hexameric structures of the archaeal secretion ATPase GspE and implications for a universal secretion mechanism. EMBO J., 26, 878-90.

Zhou,Q. et al. (2015) Architecture of the synaptotagmin-SNARE machinery for neuronal exocytosis. Nature, 525, 62-67. 
bioRxiv preprint doi: https://doi.org/10.1101/500397; this version posted January 8,2019 . The copyright holder for this preprint (which was not certified by peer review) is the author/funder. All rights reserved. No reuse allowed without permission.

van Zundert,G.C.P. et al. (2016) The HADDOCK2.2 web server: User-friendly integrative modeling of biomolecular complexes. J. Mol. Biol., 428, 720-725. 